\title{
A Simple Method for Coating Polypropylene with Gold Nanoparticles
}

Yenisey Ponce de León ${ }^{1}$, Juan Luis Pichardo-Molina ${ }^{1}$, Pablo Eduardo Cardoso-Avila ${ }^{1}$ and Monica Monserrat Martinez-Garcia ${ }^{1}$.

1. Centro de Investigaciones en Óptica, León, Guanajuato, México.

The formation of monolayer ensembles of colloidal nanoparticles (NPs) on different kinds of substrates has been drawing a lot of attention due to the fact that such nanostructured ensembles possess unique physical and chemical properties which depend on the NPs size and shape, as well as on the inter-particle distance. These systems are quite promising for applications on catalysis, microelectronics, bio-sensing and bio-imaging, as well as antibacterial surfaces [1]. Some of the most recurrent procedures for the formation of colloidal monolayers are drop-casting, dip-coating, sample immersion and transferring of densely packed ensembles of metal NPs from liquid surface by means of the LangmuirBlodgett technique [2]. Generally, the surface of the substrate needs to be treated before NPs can be deposited. UV plasma cleaning followed by silanization with organosilane molecules is one of the most common procedures, but it is laborious and time consuming [3]. On the other hand, polypropylene (PP) is currently one of the most utilized polymers in industrial processes (textile, food packing, automotive and electronics) due to its remarkable chemical and physical properties, which make it suitable to replace glass, metals, cartons and other polymers. In this work, we propose a simple method to deposit a monolayer of $\mathrm{Au}$ NPs onto the surface of PP centrifuge tubes by pouring the colloidal solutions onto the substrate.

NPs were prepared based on the classical Turkevich/Frens method with slight modifications [4], following the three steps described next. Step 1: A solution of $2.2 \mathrm{mM}$ sodium citrate in Milli-Q water $(50 \mathrm{ml})$ was heated to its boiling point, and at thame, $330 \mu \mathrm{l}$ of $25 \mathrm{mM} \mathrm{HAuCl}{ }_{4}$ solution were added; once the solution turned ruby red in color, the colloid was cooled in the same vessel until it reached 90C. Step 2: $330 \mu \mathrm{L}$ of a $\mathrm{HAuCl}_{4}$ solution $(25 \mathrm{mM})$ were injected and left to react for $30 \mathrm{~min}$. This process was repeated twice. Step 3: The sample was diluted by extracting $18.5 \mathrm{~mL}$ of sample and adding $17.7 \mathrm{~mL}$ of Milli-Q water and $800 \mu \mathrm{L}$ of $60 \mathrm{mM}$ sodium citrate. This solution was then used as a seed solution, and the steps 2 and 3 were repeated three more times to complete four growths. UV-Vis absorption spectra were acquired with a portable spectrometer and normalized with its maximum of absorbance; figure 1a shows the red shift behavior of the plasmon band located at 521, 523, 525.5 and $530.5 \mathrm{~nm}$, respectively for each growth, which means that nanoparticles are becoming bigger in each growth. Figures 1b-e show the scanning electron microscopy analysis (SEM, JEOL, JSM-7800F, operating at $1 \mathrm{kV}$ ); the calculated mean sizes of the Au NPs were $31 \pm 4,39 \pm 4,46 \pm 4$, and $54 \pm 5 \mathrm{~nm}$, respectively for each growth. For nanoparticle deposition, $15 \mathrm{ml}$ of each growth solution from step 3 of the synthesis were poured into centrifuge tubes immediately after each extraction; 24 hours later, the colloids were emptied from the tubes, and the tubes washed with plenty of Milli-Q water several times, and finally, were left to dry. Through visual inspection, a red hue was observed on the inner surface of the tubes. PP substrates were cut out in pieces of $3 \times 3 \mathrm{~mm}$ for SEM analysis. Figure 2 shows the SEM images corresponding to the substrates for each growth; 
our preliminary results indicate that a single layer of Au NPs can be formed on the PP surface with very low aggregation. Using the open source software ImageJ, we calculated the density of the deposited NPs; the corresponding determined values were 14, 47, 19 and $26 \mathrm{NPs} / \mu \mathrm{m}^{2}$ for first to fourth growth, respectively. It seems that the second growth shows the best efficiency to coat PP surfaces. Here, we have presented a simple methodology to deposit spherical Au NPs of different sizes onto the surface of PP substrates, with the advantage that PP does not require any pre-treatment.

[1] A. Inberg et al., Microelectronic Engineering, 98 (2012), p. 570.

[2] J. Kim et al., Bioconjugate Chem., 28 (2017), p. 1581.

[3] K. Fujiwara et al., Anal Sci 25 (2009), p. 241.

[4] N. G. Bastús et al., Langmuir 27(2011), p. 11098.

[5] The authors are grateful to CONACyT, Maria Christina Albor (CIO) for her technical assistance and Mario Ruiz Berganza (CIO) for proofreading the manuscript.
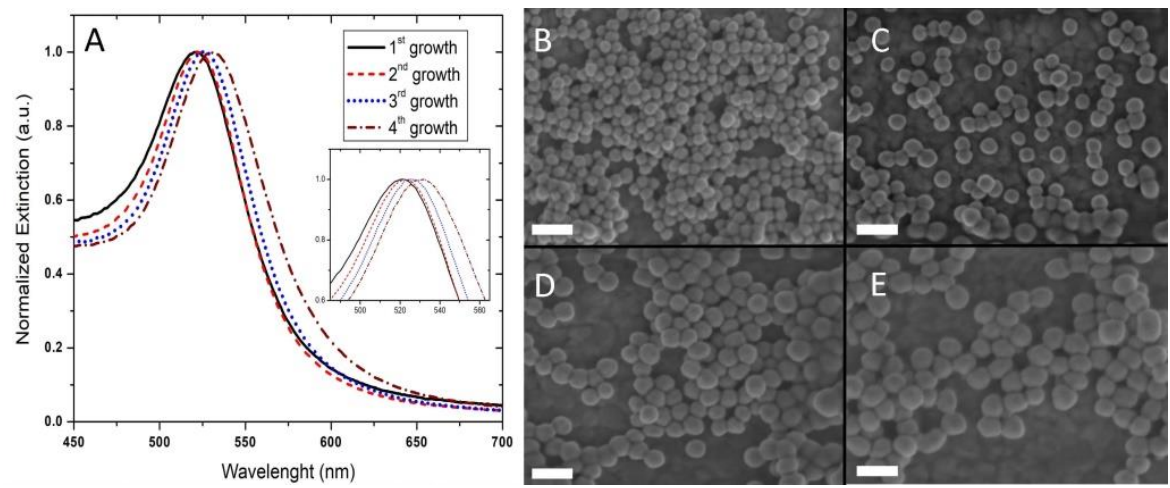

Figure 1. A) UV-Vis absorption spectra of the fourth growths. B) - E) SEM images of the spherical gold NPs synthesized, first to fourth growth respectively. Scale bar is $100 \mathrm{~nm}$.

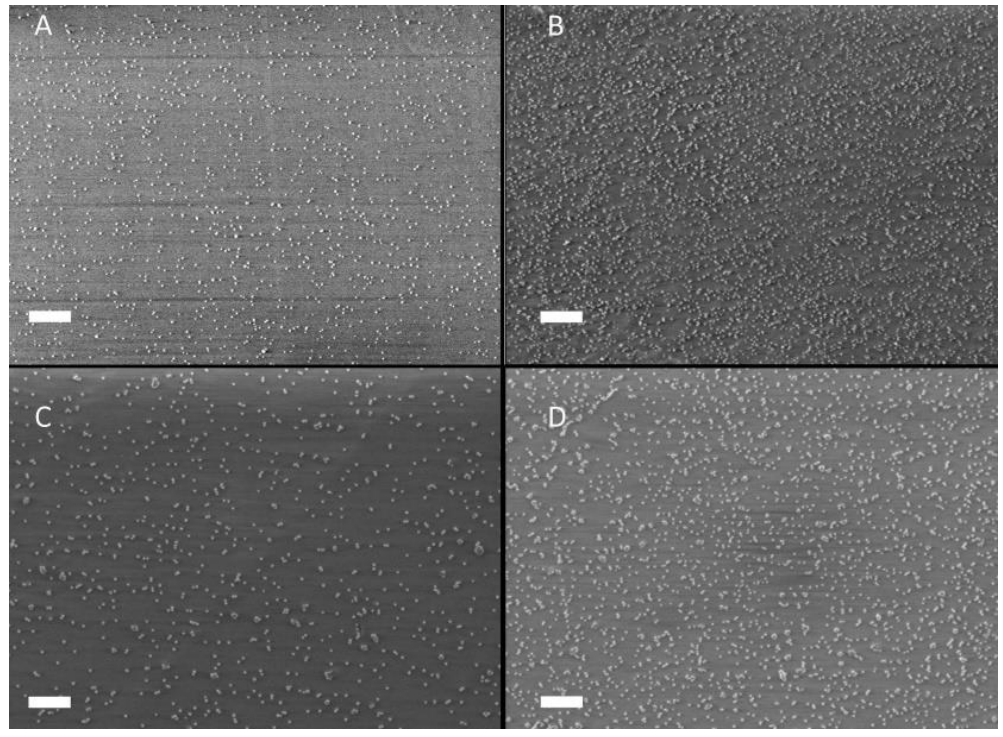

Figure 2. SEM images of spherical gold NPs on the polypropylene substrates (A - D, first to fourth growth respectively). Scale bar is $1 \mu \mathrm{m}$. 